\title{
ON POINTS OF JACOBIAN RANK $k$. II
}

\author{
P. T. $\mathrm{CHURCH}^{1}$
}

In this paper the following theorem is proved:

TheOREM 1. Let $f: M^{n} \rightarrow N^{p}$ be $C^{r}$, where $M^{n}$ and $N^{p}$ are $C^{r}$ manifolds, and let $R_{k}(f)$ be the set of points in $M^{n}$ at which the Jacobian matrix of $f$ has rank at most $k(0 \leqq k \leqq \min (n, p))$. Let $i_{*}: \pi_{m}\left(N^{p}-f\left(R_{k}\right)\right) \rightarrow \pi_{m}\left(N^{p}\right)$ be the homomorphism on the mth homotopy groups induced by the inclusion map $i$. Then $i_{*}$ is an isomorphism (onto) for $m+k \leqq p-2$ and $r \geqq \max (n-p+m+2,1)$, and is onto for $m+k \leqq p-1$ and $r \geqq \max (n-p+m+1,1)$.

In the previous paper [3] this theorem was proved except that the hypothesis " $C^{n}$ ", rather than " $C^{r}$, where $r \geqq \max (n-p+m+2,1)$ " etc., was used. Besides improving the differentiability hypothesis, the present proof is shorter, and shows the connection between this theorem and a theorem of Thom $[10$, p. 26]. On the other hand, this proof does not yield $[3,(1.2)]$, whose proof requires almost all the lemmas of that paper.

From the examples of $[3$, p. $421,(3.3)]$ it follows that the differentiability hypotheses of Theorem 1 are the best possible for all $n, p$, and $m$ with $0 \leqq p-n \leqq m$. A new proof of $[2$, p. 88, (1.3)] is also given (Proposition 4).

Manifolds in this note are separable, without boundary, but not necessarily connected.

REMARK 2. In his proof of $[10$, p. 26, Theorem I.5] Thom used a theorem of A. P. Morse [10, p. 20]; if Sard's Theorem [9] is used instead, the differentiability hypothesis can be changed from $C^{n}$ to $C^{\max (n-q+1,1)}$. Also, in [10, Theorem I.6] the hypothesis $C^{1}$ suffices.

Furthermore, if $r$ is any positive integer and $H$ on p. 22 of [10] is the group of $C^{r}$ diffeomorphisms rather than $C^{n}$, then Thom's proof of Theorem I.5 actually yields $A$ a $C^{r}$ diffeomorphism.

The following lemma is essentially $[3$, p. 419, (3.2)] with improved differentiability hypotheses.

Lemma 3. Let $f: M^{n} \rightarrow N^{p}$ be a $C^{r}$ map, where $M^{n}$ and $N^{n}$ are $C^{r}$ manifolds, let $k$ be an integer with $0 \leqq k \leqq \min (n, p)$, let $Q$ be a finite polyhedron with $\operatorname{dim} Q \leqq p-1-k$, and let $r \geqq \max (n-p+\operatorname{dim} Q+1,1)$. Let $\epsilon$ be positive, let $\Delta \subset Q$ be a subpolyhedron, and let $\alpha: Q \rightarrow N^{p}$ be a

Received by the editors July 10, 1964.

${ }^{1}$ Research supported in part by National Science Foundation Grant GP 2193. 
map such that $\alpha(\Delta) \cap f\left(R_{k}(f)\right)=\varnothing$. Then there exists a map $\gamma: Q \rightarrow N^{p}$ such that $\gamma(Q) \cap f\left(R_{k}(f)\right)=\varnothing, \alpha|\Delta=\gamma| \Delta$, and the uniform distance $d(\alpha, \gamma)<\epsilon$.

Proof. We may suppose that $M^{n}$ and $N^{p}$ are $C^{\infty}$ manifolds [6, p. 41]. By the Whitney Embedding Theorem $N^{p}$ has a complete Riemannian metric $g$, and we may as well suppose that its given distance function $d$ is that induced by $g[5$, p. 166, (3.5)].

Let $Y$ be any compact set in $M^{n}$; we first prove the lemma for $R_{k}(f)$ replaced by $Y \cap R_{k}(f)$. Let $\mu=d\left(\alpha(\Delta), f\left(Y \cap R_{k}(f)\right)\right)$, and let $V=\left\{x \in N^{p}: d(x, \alpha(\Delta))<\mu / 2\right\}$. We may suppose that $\epsilon<\mu / 2$. Since $\bar{V}$ is compact [5, p. 172], there exists $\nu, 0<\nu<\epsilon$, such that for each $z \in \bar{V}$ the open ball $U(z, \nu)$ with center $z$ and radius $\nu$ is that given by $[5$, p. $149,(8.7)]$. There exists an open neighborhood $W$ of $\Delta$ such that $\bar{W} \neq Q$ and $\alpha(\bar{W}) \subset V$.

We will define a map $\beta: Q \rightarrow N^{p}$ such that $d(\alpha, \beta)<\nu$ and $\beta(Q) \cap f\left(Y \cap R_{k}(f)\right)=\varnothing$. The manifold $N^{p}$ has a triangulation induced by the differential structure $\left[6\right.$, p. 101, (10.6)]; let $\delta: Q \rightarrow N^{p}$ be a simplicial approximation to $\alpha$ with $d(\alpha, \delta)<\nu / 2$. Let $\Gamma_{i}(i=1,2, \cdots, s)$ be the (open) simplices of the polyhedron $\delta(Q)$, in order of increasing dimension.

If $I$ is the identity diffeomorphism on $N^{p}$, there is $([10$, p. 26] and Remark 2) a $C^{r}$ diffeomorphism $A$ of $N^{p}$ onto itself such that the uniform distance $d(A, I)<\nu / 4$ and $f$ is transverse regular $[10$, p. 22] on $A^{-1}\left(\Gamma_{1}\right)$. Since $f$ has rank at least $p-\operatorname{dim}\left(\Gamma_{1}\right)(=p)$ at each point of $f^{-1}\left(A^{-1}\left(\Gamma_{1}\right)\right)$ (it may be empty), and $k+1 \leqq p-\operatorname{dim} Q$, $A^{-1}\left(\Gamma_{1}\right) \cap f\left(R_{k}(f)\right)=\varnothing$. Since a $C^{r}$ diffeomorphism may be approximated by a $C^{\infty}$ diffeomorphism $\left([6\right.$, p. $39,(4.5)]$; the $f_{1}$ in $(4.3)$ and (4.5) may be chosen to approximate $f$ ), there is a $C^{\infty}$ diffeomorphism $B$ such that $d(B, I)<\nu / 4$ and $B^{-1}\left(\Gamma_{1}\right) \cap f\left(Y \cap R_{k}(f)\right)=\varnothing$. Let $\Psi_{1}=B^{-1}$.

We continue by induction. Suppose that a $C^{\infty}$ diffeomorphism $\Psi_{i}$ of $N^{p}$ onto itself has been defined such that

$$
\left(\mathfrak{P}_{i}\right) d\left(\Psi_{i}, I\right)<\left(2^{-1}-2^{-i-1}\right) \nu \text { and } \Psi_{i}\left(\bigcup_{j=1}^{i} \Gamma_{j}\right) \cap f\left(Y \cap R_{k}(f)\right)=\varnothing \text {. }
$$

Choose $\xi$ such that $0<\xi<2^{-i-2} \nu$ and (since $\bigcup_{j=1}^{i} \Gamma_{j}$ is compact)

$$
\xi<d\left(\Psi_{i}\left(\bigcup_{j=1}^{i} \Gamma_{j}\right), f\left(Y \cap R_{k}(f)\right)\right)
$$

As above, there is a $C^{r}$ diffeomorphism $A$ of $N^{p}$ on to itself such that $d(A, I)<\xi$ and $f$ is transverse regular on $A^{-1}\left(\Psi_{i}\left(\Gamma_{i+1}\right)\right)$. Again 


$$
A^{-1}\left(\Psi_{i}\left(\Gamma_{i+1}\right)\right) \cap f\left(Y \cap R_{k}(f)\right)=\varnothing,
$$

and we may suppose that $A$ is $C^{\infty}$. Let $\Psi_{i+1}=A^{-1} \Psi_{i} ; \Psi_{i+1}$ satisfies condition $\mathfrak{P}_{i+1}$. The map $\beta=\Psi_{8} \delta$ satisfies the desired conditions: $d(\alpha, \beta)<\nu$ and $\beta(Q) \cap f\left(Y \cap R_{k}(f)\right)=\varnothing$.

For each $x \in \bar{U}, \beta(x) \in V(\alpha(x), \nu)$. Let $t$ be the continuous real valued function defined on $Q$ by

$$
t(x)=d(x, \Delta)[d(x, \Delta)+d(x, Q-W)]^{-1} .
$$

For each $x \in W$, let $\gamma(x)$ be the (unique) point on the geodesic joining $\alpha(x)$ to $\beta(x)$ in $U(\alpha(x), \nu)[5$, p. 166, Theorem 3.6] such that

$$
t(x)=d(\alpha(x), \gamma(x))[d(\alpha(x), \beta(x))]^{-1} ;
$$

for each $x \in Q-W$, let $\gamma(x)=\beta(x)$. It follows from the proof of [5, p. 150, Lemma 2] that $\gamma: Q \rightarrow N^{p}$ is continuous (if $\phi$ is the diffeomorphism of that lemma, then $\gamma(x)=\exp \left(t(x) \cdot \phi^{-1}(\alpha(x), \beta(x))\right)$, where - is scalar multiplication in $\left.E^{p}\right)$. Since $t=0$ on $\Delta, \gamma|\Delta=\alpha| \Delta$; since $\gamma|(Q-W)=\beta|(Q-W), \quad \gamma(Q-W) \cap f\left(Y \cap R_{k}(f)\right)=\varnothing$. Since $\gamma(x) \in U(\alpha(x), \nu)$ for each $x \in W$, and $\nu<\epsilon<\mu / 2, d(\alpha(x), \gamma(x))<\mu / 2$ for each $x \in W$; and since $\alpha(\bar{W}) \subset V, d(\alpha(x), \alpha(\Delta))<\mu / 2$ also. It follows that $\gamma(W) \cap f\left(Y \cap R_{k}(f)\right)=\varnothing$. This completes the proof in case $R_{k}(f)$ is replaced by $Y \cap R_{k}(f)$, where $Y$ is any compact subset of $M^{n}$.

The manifold $M^{n}=\bigcup_{j=1}^{\infty} Y_{j}$, where $Y_{j} \subset Y_{j+1}$ and $Y_{j}$ is compact. Define inductively a sequence of maps $\gamma_{j}: Q \rightarrow N^{p}\left(\gamma_{0}=\alpha\right)$ such that

$$
d\left(\gamma_{j}(Q), f\left(Y_{j} \cap R_{k}(f)\right)\right)>0
$$

(call it $\left.\eta_{j}\right), \quad \gamma_{j}\left|\Delta=\gamma_{j-1}\right| \Delta$, and $d\left(\gamma_{j}, \gamma_{j-1}\right)<2^{-j \zeta}$, where $\zeta<\min \left(\epsilon, \eta_{i}\right)(i=1,2, \cdots, j-1 ; j=1,2, \cdots)$. Since $N^{p}$ is a complete metric space, the limit of the $\gamma_{j}$ exists, call it $\gamma$; it is the desired map. To prove that $\gamma(Q) \cap f\left(R_{k}(f)\right)=\varnothing$, one observes that $\gamma(Q) \cap f\left(Y_{j} \cap R_{k}(f)\right)=\varnothing(j=1,2, \cdots)$.

Theorem 1 is an easy consequence of Lemma 3 [3, p. 421].

The following statement was originally proved by the author $[2$, p. $88,(1.3)]$ under the hypothesis $C^{n}$, and then by Sard $[8, \S 5$, Theorem 2] under the present hypothesis.

Proposition 4. If $M^{n}$ and $N^{p}$ are $C^{\max (n-k, 1)}$ manifolds, and $f: M^{n}$ $\rightarrow N^{p}$ is $C^{\max (n-k, 1)}$, then $\operatorname{dim}\left(f\left(R_{k}(f)\right)\right) \leqq k$. In particular, if $M^{n}$ and $N^{n}$ are $C^{1}$ manifolds and $f: M^{n} \rightarrow N^{n}$ is $\bar{C}^{1}$, then $\operatorname{dim}\left(f\left(M^{n}\right)\right) \leqq n$.

We now observe that this theorem can also be obtained from Thom's theorem $[10$, p. 26].

Proof. We may suppose that $N^{p}=E^{p}$, and prove that $\operatorname{dim}\left(f\left(Y \cap R_{k}(f)\right)\right) \leqq k$, where $Y$ is any compact subset of $M^{n}$. Suppose 
the contrary, i.e., $\operatorname{dim}\left(f\left(Y \cap R_{k}(f)\right)\right) \geqq k+1$. There exists a compact subset $C$ of $f\left(Y \cap R_{k}(f)\right)$ such that the homomorphism

$$
i^{*}: H^{k}\left(f\left(Y \cap R_{k}(f)\right) ; Z\right) \rightarrow H^{k}(C ; Z)
$$

(of the Cech cohomology groups with integer coefficients) induced by inclusion is not onto [4, p. 151]. The Alexander Duality Theorem [1, p. 52] applied separately to $f\left(Y \cap R_{k}(f)\right)$ and $C$ yields a homomorphism

$$
j_{*}: H_{p-k-1}\left(E^{p}-f\left(Y \cap R_{k}(f)\right) ; Z\right) \rightarrow H_{p-k-1}\left(E^{p}-C ; Z\right)
$$

(of the Cech homology groups with compact support-augmented in dimension zero) which is also not onto. Moreover $j_{*}$ is induced by inclusion. (The author is grateful to $F$. Raymond for verifying this fact; cf. also $[7, \S 5]$.)

Let $z$ be a polyhedral cycle of $E^{p}-C$ whose homology class $\gamma$ is not in the range of $j_{*}$, and let $\Gamma$ be the carrier of $z$. Applications of $\left[10\right.$, p. 26] as in Lemma 3 yield a cycle $y \in \gamma$ with carrier $A^{-1}(\Gamma)$ disjoint from $f\left(Y \cap R_{k}(f)\right)$. Thus $\gamma$ is in the range of $j_{*}$ and a contradiction results.

There is a shorter and more natural proof by induction using Thom's theorem and the inductive definition of dimension; unfortunately it requires the hypothesis $C^{n}$.

\section{REFERENCES}

1. P. S. Aleksandrov, Combinatorial topology, Vol. 3, Graylock Press, Albany, N. Y., 1960.

2. P. T. Church, Differentiable open maps on manifolds, Trans. Amer. Math. Soc. 109 (1963), 87-100.

3. - On points of Jacobian rank k, Trans. Amer. Math. Soc. 110 (1964), 413-423.

4. W. Hurewicz and H. Wallman, Dimension theory, Princeton Univ. Press, Princeton, N. J., 1941.

5. S. Kobayashi and K. Nomizu, Foundations of differential geometry, Vol. I, Interscience, New York, 1963.

6. J. R. Munkres, Elementary differential topology, Princeton Univ. Press, Princeton, N. J., 1963.

7. F. Raymond, Local cohomology groups with closed supports, Math. Z. 76 (1961), $31-41$.

8. A. Sard, Hausdorf measure of critical images on Banach manifolds, Amer. J. Math 87 (1965), 158-174.

9. - The measure of the critical values of differentiable maps, Bull. Amer. Math. Soc. 48 (1942), 883-890.

10. R. Thom, Quelques propriêtés globales des variêtés différentiables, Comment. Math. Helv. 28 (1954), 17-86.

SYracuse UNIVERSITY 